\title{
Kennedy Terminal Ulcers
}

\section{A Scoping Review}

\author{
Sharon Latimer, PhD \\ Kristyn Mackrell, BSc O \\ Joanie Shaw, GradCert \\ Brigid M. Gillespie, PhD
}

O Tracey Hunt, MSN O

Kennedy terminal ulcers, a subset of pressure injuries, are associated with the dying process. This scoping review aimed to identify and map the published literature on Kennedy terminal ulcers in terms of its definition, prevalence, assessment, treatment, management, health care costs, and quality of life for patients in all health care settings. Using the Arksey and O'Malley scoping review framework, we systematically searched the Cochrane Library, CINAHL, EMBASE, MEDLINE, and ProQuest databases and 5 guideline repositories between 1983 and 2018. The following search terms were used: Kennedy ulcers, Kennedy terminal ulcers, terminal ulcer, skin failure, and Skin Changes at Life's End. Data were extracted using a purposely developed data collection tool. Initial searches yielded 2997 sources, with 32 included in this review. Most Kennedy terminal ulcer literature was published by nurses in the United States. Kennedy terminal ulcer prevalence data are limited, with no validated assessment tools available. Kennedy terminal ulcers may be misclassified as pressure injuries, potentially resulting in financial penalties to the institution. This scoping review revealed significant knowledge and clinical practice gaps in patient assessment, management, and treatment of Kennedy terminal ulcers. Timely patient education may help them to make informed care and quality end-of-life decisions. Further research is needed to inform clinical practice to improve patient care.
Sharon Latimer, PhD, is research fellow (Patient Safety in Nursing), School of Nursing and Midwifery, Griffith University; and Nursing Midwifery Education and Research Unit, Gold Coast Hospital and Health Service, Southport, Queensland, Australia.

Joanie Shaw, GradCert, is nurse educator, Cancer and Specialty Services, Gold Coast Hospital and Health Service, Southport, Queensland, Australia.

Tracey Hunt, MSN, is clinical nurse consultant, Diagnostics and Subspecialty Services, Gold Coast Hospital and Health Service, Southport, Queensland, Australia.

Kristyn Mackrell, BSc, is senior occupational therapist, Community \& Palliative Care Occupational Therapy Services, Gold Coast Hospital and Health Service, Southport, Queensland, Australia.

Brigid M. Gillespie, PhD, is professor (Patient Safety in Nursing), graduate certificate, School of Nursing and Midwifery, Griffith University; and Nursing Midwifery Education and Research Unit, Gold Coast Hospital and Health Service, Southport, Queensland, Australia.

\section{KEY WORDS}

acute care, end of life, hospice, palliative care, wounds

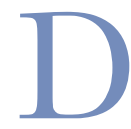
ying patients have specific clinical care requirements. In the days and/or weeks prior to their death, some of these patients develop a skin integrity breach known as a Kennedy terminal ulcer (KTU). ${ }^{1,2}$ First observed and described in the United States in 1989, KTUs are a subset of pressure injuries (PIs) not easily recognized by clinicians. ${ }^{3-5}$ This can influence their accurate diagnosis and management and impact patients in terms of pain and comfort. ${ }^{3-5}$ Recently, consensus was reached concerning the unavoidable nature of KTUs among some dying patients ${ }^{3,6}$ and clinicians' use of pressure-relieving equipment. ${ }^{7}$ Hence, additional research is needed to help clinicians to better understand KTUs and find ways to provide patients with greater treatment options. ${ }^{4,5,8}$

Scoping reviews are useful to map the published literature on topics where previous reviews are lacking or where a dearth of literature exists. ${ }^{9,10}$ These reviews permit researchers and clinicians to identify and describe knowledge gaps, providing a useful guide for future clinical research. ${ }^{9,10}$ The aims of this scoping review were to identify published literature on KTUs among all patients in acute care, hospice, and nursing home settings and to provide an analytical synthesis of the findings.

This research did not receive any specific grant from funding agencies in the public, commercial, or not-for-profit sectors.

The authors conceived and designed this review. The database searches were undertaken by S.L. and J.S. Literature screening and eligibility were completed by S.L., J.S., and B.M.G. Data extraction was carried out by S.L., T.H., and K.M. Manuscript drafting was led by S.L. and B.M.G. All authors made substantial manuscript contributions and approved the final submitted draft.

The authors have no conflicts of interest to disclose.

Address correspondence to Sharon Latimer, PhD, School of Nursing and Midwifery, Logan Campus, Griffith University, L05 3.44, Meadowbrook, Queensland, Australia 4131 (s.latimer@griffith.edu.au).

Supplemental digital content is available for this article. Direct URL citations appear in the printed text and are provided in the HTML and PDF versions of this article on the journal's Web site (www.jhpn.com).

Copyright (C) 2019 by The Hospice and Palliative Nurses Association. All rights reserved.

DOI: 10.1097/NJH.0000000000000563 


\section{METHODS}

Arksey and O'Malley's 9 scoping review methodology was followed because it allows researchers and clinicians to explore the breadth of published literature on health care topics ${ }^{11,12}$ regardless of the study design. ${ }^{9}$ Prior to undertaking this review, we developed an a priori study protocol using the Preferred Reporting Items for Systematic Reviews and Meta-analyses Extension for Scoping Reviews checklist. ${ }^{13}$

The 5-step Arksey and O'Malley's scoping review framework includes (1) identifying research questions; (2) locating relevant records via various sources; (3) selecting appropriate literature and/or studies; (4) data charting; and (5) collating, summarizing, and reporting the results. 9 The quality of the identified sources was not appraised ${ }^{9}$ because most were not original research, with our aim focused on examining the breadth of the KTU literature.

\section{Step 1: Research Questions}

We posed the following research questions:

1. How is KTU defined?

2. What is the prevalence of KTUs in acute care, hospice, and nursing home settings?

3. How are KTUs assessed and what risk assessment tools are used in acute care, hospice, and nursing home settings?

4. How are KTUs managed and treated in acute care, hospice, and nursing home settings?

5. What are the health care costs associated with the management and treatment of KTUs in acute care, hospice, and nursing home settings?

6. What are the quality of life (eg, pain) and psychological impacts on patients with KTUs in acute care, hospice, and nursing home settings?

For this review, an acute care setting is defined as a hospital where patients' medical and surgical clinical conditions are diagnosed, treated, and managed. ${ }^{14}$ Hospice care, delivered in hospitals, palliative care units, communities, or nursing home settings, is defined as the end-of-life care provided to those with a terminal illness. ${ }^{15,16}$ Nursing home settings provide long-term care to mainly older patients, with the terms aged-care facility, long-term-care facility, assisted-living facility, and care home used in the literature interchangeably with the term nursing bome. ${ }^{17}$

\section{Step 2. Locating Relevant Literature via Various Sources}

A systematic database and gray literature search strategy was developed. The search terms used were Kennedy ulcer, Kennedy terminal ulcer, terminal ulcer, skin failure, and Skin Changes at Life's End (SCALE). No synonyms or PubMed database mesh terms were identified for the search terms. Quotation marks were placed around search terms, and Boolean operators (OR, AND) were used. Database search publication limiters of January 1982 to October 2018 were applied because Kennedy's ${ }^{1}$ seminal KTU study commenced in 1983.

On December 21, 2017, and October 20, 2018, PubMed, CINAHL Plus, MEDLINE, ProQuest Central, EMBASE, Scopus, Web of Science, and the Cochrane Library databases were searched. In December 2017 and June 2018, the gray literature was searched via OpenGrey, Google Scholar, Trove (National Library of Australia), Agency for Healthcare Research and Quality, National Guideline Clearinghouse, Australian Clinical Practice Guidelines, BMJ clinical evidence, Australian Institute of Health and Welfare, BMC proceedings, EThOS, Analysis and Policy Observatory, WorldCat, and a domain search (.gov.au). The reference lists of identified reviews and dissertations were manually examined for additional relevant sources.

\section{Step 3. Study Selection}

Inclusion and exclusion criteria for literature selection were developed and revised. ${ }^{9}$ We used broad inclusion criteria that included dying patients, adults and pediatric patients, all health care settings, articles published in English, primary research articles regardless of methodology, case studies, clinical practice guidelines, standards/documents from professional organizations, websites, newspapers, and conference posters. Pressure ulcer/injury and PI prevention publications were excluded. Initial identified references were imported into Endnote (version 8; Clarivate Analytics, Philadelphia, Pennsylvania), a citation management software. Following duplicate removal, 2 researchers (S.L., J.S.) screened the titles and abstracts, assigning the following ratings: include, exclude, and adjudicate. A third reviewer (B.M.G.) adjudicated when there was a lack of consensus and made the final decision on their inclusion. Using the study inclusion/exclusion criteria, the final selected full-text articles and gray literature were screened (S.L., J.S.) and adjudicated (B.M.G.).

\section{Step 4. Data Charting}

We pilot tested the Microsoft Excel data extraction tool using 2 identified full-text articles and made minor tool adjustments (eg, layout). Three researchers (S.L., T.H., K.M.) independently extracted the data using this tool.

\section{Step 5. Collating, Summarizing, and Reporting the Results}

Our findings are collated and synthesized quantitatively and narratively relative to the extent, nature, and distribution of the identified sources ${ }^{9}$ and in relation to our research questions.

\section{RESULTS}

A total 2997 sources were initially identified from the databases and gray literature, with no additional sources found 
in the reference lists of relevant KTU reviews and dissertations. Following screening and eligibility, 32 journal and gray literature sources were included in this scoping review. Supplemental Digital Content 1 (http://links.lww. $\mathrm{com} / \mathrm{JHPN} / \mathrm{A} 31$ ) outlines the study selection process following the Preferred Reporting Items for Systematic Reviews and Meta-analyses. ${ }^{18}$

\section{Publication Characteristics}

Of the 32 KTU scoping review sources, most are published in health care journals (Supplementary Digital Content 2, http://links.lww.com/JHPN/A32). One-quarter ( $=$ 8 [25\%]) of the sources were peer reviewed ${ }^{1,4,5,8,19-22}$ and used either observational quantitative or qualitative methodologies.

Almost two-thirds (65.6\%) of the scholarly sources solely focused on KTU (Supplementary Digital Content 3, http://links.lww.com/JHPN/A33), with the remainder including KTU information such as definitions and treatment, as part of a broader discussion on general wound care, skin failure, SCALE, and palliative care. In 1989, Kennedy ${ }^{1}$ was the first to publish information on KTU. Since then, there has been on average 1 new KTU publication per year, with an average of 5 new sources per year over a 6-year period (2010-2016). Most of the sources $(n=26[81.3 \%])$ originated from the United States $^{1-5,8,16,19-37}$ and were authored by nurses $(n=22$ [69\%]).

Across the $10 \mathrm{KTU}$ research studies, 878 participants were recruited, $1,5,8,19,26,30,32-35$ which included patients and nurses, with sample sizes ranging from 1 to 469. Apart from 1 pediatric ( 5 -month-old) participant, ${ }^{32}$ the remaining recruited participants were aged between 15 and 104 years. Only 1 study explored nurses' perceptions $(n=13)$ of caring for patients with end-of-life wounds, including KTU. ${ }^{8}$ No authors reported research funding support. These KTU research studies were conducted in hospices $(\mathrm{n}=3[27.3 \%]),{ }^{5,8,30}$ long-term care facilities $(\mathrm{n}=2$ $[18.2 \%]),{ }^{34,35}$ the community $\left(\mathrm{n}=1\right.$ [9.1\%]), ${ }^{26}$ an intermediate care facility $(n=1[9.1 \%]),{ }^{1}$ acute care $(n=1[9.1 \%]),{ }^{33}$ palliative care $(n=1[9.1 \%]),{ }^{19}$ and a pediatric unit $(n=1$ [9.1\%]). ${ }^{32}$ A 2012 study of 80 participants undertaken by Trombley et $\mathrm{al}^{20}$ is included in this scoping review because the article contained general KTU information. A new phenomenon named Trombley-Brennan terminal tissue injuries (TB-TTIs) is described, which the authors assert is different from KTU. ${ }^{20}$ Trombley-Brennan terminal tissue injuries are a type of tissue injury now recognized by the National Pressure Ulcer Advisory Panel (NPUAP) ${ }^{38,39}$ and are defined in the Discussion.

\section{Definitions of KTU}

Kennedy terminal ulcer was defined in 26 sources. ${ }^{1-8,16,19,21-23,}$ $25-28,33-37,40-43$ Skin failure is a term used to describe skin changes at the end of life, which include KTU, SCALE, TB-TTIs, and Charcot decubitus ominosus. ${ }^{3,33}$ These terms are used interchangeably to describe skin changes at life's end, ${ }^{33}$ increasing the confusion across the literature. In 1877 , Charcot first documented butterfly-shaped lesions on the buttocks of dying patients. ${ }^{43}$ A century later, Kennedy ${ }^{1}$ described KTU as a subset of PI, ${ }^{7,16}$ originally naming them Kennedy terminal lesion. Renamed KTU in 1989, there are 2 agreed presentations: bilateral and unilateral. ${ }^{1-3}$ Bilateral KTU is a butterfly-, horseshoe-, or pear-shaped lesion, with irregular margins. ${ }^{1,3}$ The lesion suddenly appears on the patient's sacrum or coccyx, 2 weeks to several months prior to their death. ${ }^{1,3}$ First appearing as erythematous and/or purpuric, bilateral KTUs then progress to a yellow and/or black color, with the presence or absence of epidermal erosion. ${ }^{1,3}$ Unilateral KTU, also known as "3:30 syndrome," is a macular lesion of less than $1 \mathrm{~cm}^{2}$, which is either purpuric or has irregular black margins and appears on only 1 buttock. $^{1,3}$ This rapidly developing lesion is seen 8 to 24 hours prior to death, with the absence of epidermal erosion. ${ }^{1,3}$ The term "3:30 syndrome" was coined for unilateral KTU because this was often the time of day when staff observed these rapid skin changes. ${ }^{3}$

Kennedy terminal ulcers are considered unavoidable, meaning that in the presence of pressure and shear and despite a full clinical assessment including the implementation of prevention clinical care, these lesions still occur. ${ }^{3,5-7,24,34,36,37,42}$ The etiology of KTU is unknown; however, it is theorized that during the dying process skin changes occur in some patients due to end-of-life disease,

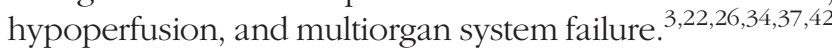
The lack of robust KTU research evidence fuels the debate around the "existence" of KTU. 16,20,29,31,33,36,43 In 2008, an expert panel concluded there is limited knowledge about SCALE wounds ${ }^{7,27}$ including factors that may increase a patient's likelihood of KTU development ${ }^{40}$ such as malnutrition ${ }^{41}$ and immobility. ${ }^{16}$ However, based on the evidence available at the time, the SCALE panel developed a consensus statement acknowledging KTU as an unavoidable phenomenon that, despite appropriate care, can occur in the period prior to death. ${ }^{3,36}$ The NPUAP endorsed this statement in 2010, adding that removing pressure from tissues does not guarantee skin survival. $^{3}$

Despite the NPUAP's endorsement, ${ }^{3}$ the 3 authors challenge the hypothesized etiology of KTU, ${ }^{20,29,31}$ with 2 offering new tissue injury concepts: TB-TTIs and Miller pressure equivalent injuries. $^{20,29}$ In 2010, Olshansky ${ }^{31}$ suggested unavoidable KTUs result from inadequate care and pressure relief and not the dying process. In her retort, Yastrub, a legal defense expert witness, states "pressure ulcer is a diagnosis of exclusion, not the other way around." 31(p466) She adds, that because of a lack of awareness and research, many health care professionals 
incorrectly diagnose KTU as PI. ${ }^{31}$ Following on from Brennan and Trombley's ${ }^{19}$ published KTU study in 2010, Trombley et $\mathrm{al}^{20}$ published another descriptive study of 80 palliative care patients in 2012. During their chart review of these patients with end-of-life disease with tissue alterations prior to their death, the researchers proposed a new phenomenon called TB-TTIs. ${ }^{20}$ When describing TB-TTIs, the authors state that regardless of aggressive repositioning and strict adherence to wound protocols, 79 of their participants at the time of their death developed unstageable tissue injuries that remained intact. ${ }^{20}$ They hypothesize these TB-TTIs are unavoidable, located on body parts where there are no pressure points, and caused by endof-life organ failure, ${ }^{20}$ a position now supported by the NPUAP. ${ }^{38,39}$ In 2017, Miller $^{29}$ also refuted the cause of KTU being the dying process, suggesting these tissue injuries likely result from gaps in care. Further, Miller ${ }^{29}$ states the concept of KTU is not viable and should be discontinued because "systemic diseases have an equal effect on all body tissues," ${ }^{45)}$ and the stressor of pressure is localized. Instead, the author suggests a new concept of Miller pressure equivalent injuries, which accepts the dying process as another systemic stressor, not the main causative factor of pressure-based tissue injuries. ${ }^{29}$

\section{Prevalence of KTUs}

Ten sources, mostly case studies, outlined KTU prevalence data, ${ }^{1,5,19,21,26,30,32-35}$ with some suggesting hospice patients are most at risk. 5,7,8,21,26,28,30 Kennedy terminal ulcers are underrecognized in the pediatric population, with unknown prevalence data and only 1 published case study available. ${ }^{32}$ Between 1989 and 2016, 3 observational studies with a total of 854 participants reported adult KTU prevalence data. 1,5,19 The seminal KTU work published by Kennedy ${ }^{1}$ in 1989 was a 5-year (1983-1988) retrospective study of 469 adult patients, reporting that $55.7 \%$ of intermediate care participants (those needing ongoing medical and nursing care such as rehabilitation) died within 6 weeks of developing a PI. Following this longitudinal study, the researchers hypothesized the appearance of PI among dying patients might suggest their imminent death. ${ }^{1}$ In 2010, Brennan and Trombley ${ }^{19}$ published a 12-month observational study in their palliative care unit. They found 22 patients who developed a rapidly increasing PI also died within 2 hours and 6 days; equating to a $5 \%$ prevalence. ${ }^{19}$ In 2016 , Nesovic ${ }^{5}$ published a retrospective chart audit of 363 hospice patients to determine the prevalence of PI and KTU. The lack of detailed chart documentation prompted the researchers to use a proxy definition for KTU: the presence of sacral and coccyx ulcers following hospice admission. ${ }^{5}$ Using this proxy, a $6.1 \%$ KTU prevalence was reported ${ }^{5}$; however, caution is needed in interpretation because not all sacral and coccyx ulcers are KTUs.

\section{Risk Assessment of KTU Risk}

Nine sources described the risk assessment of KTU. ${ }^{3-5}$, 23,26,32-34,37 Assessing and staging KTU is difficult because of the lack of validated assessment tools ${ }^{3}$ and the low rates of KTU awareness among clinicians. ${ }^{5}$ Specialized clinician knowledge and skills are required in diagnosing KTU. ${ }^{3,37}$ It is recommended that only advanced clinical specialists undertake this assessment, ${ }^{26,33}$ which should include a detailed patient history, blood tests (full blood count, prealbumin levels, interleukins), identification of organ failure and vascular insufficiency, and the onset of the dying process. $^{3,37}$

The importance of an accurate KTU diagnosis helps to minimize assertions of negligence or a lack of care being leveled at health care professionals and carers. ${ }^{23,24,31,32,34,35,37}$ Seven of the identified sources highlighted that some KTUs are incorrectly assessed as hospital-acquired PI, ${ }^{23,37}$ thus attracting financial ${ }^{23,24,32}$ and professional regulatory penalties. ${ }^{4,24,31,34}$ Prior to 2008, the misdiagnosis of KTU resulted in the imprisonment of some carers for elder abuse. 4,34,37 Eliminating elder abuse as a causative factor of the presence of ulcers should be part of the assessment process. ${ }^{34,37}$ It is recommended for all dying patients that skin ulcers are initially assessed and staged as a PI, until it is determined that this diagnosis had been excluded and replaced by a KTU diagnosis. ${ }^{23,31}$ The quality of all future patient care rests solely on the accuracy of the KTU assessment and diagnosis. ${ }^{37}$

\section{Management and Treatment of KTUs}

In all, 17 sources included varied information about KTU treatment and management. ${ }^{2-8,16,22,25,26,28,32,35,37,41,42}$ One source focused on KTU in the pediatric population, with scant wound care treatment approaches offered. ${ }^{32}$ Adult patients featured in the remaining sources, with 2 describing end-of-life wound care among the elderly 8,35 and 1 examining wound care in dying adults with diabetes. ${ }^{41}$ Some suggest that KTU is managed and treated in the same manner as a PI in terms of pressure relief and wound ulcer dressings. ${ }^{22,28}$ However, caring for a patient with a KTU is complex, ${ }^{4,8}$ requiring a combined approach of palliative care, ${ }^{16,41}$ pressure-relieving skin care, ${ }^{7,16,28}$ advanced wound management, 3,16,25,32,42 wound exudate and odor management, ${ }^{6,16,25}$ pain management, ${ }^{2,6,8,25}$ and family and staff education and counseling. 5,7,16,25,26,35,37

Consultation with specialist wound nurses, physicians, and allied health care professionals is the first step in KTU management, which begins with the correct diagnosis. ${ }^{16,25,42}$ An interdisciplinary health care team should be assembled to ensure an appropriate care plan is developed that incorporates the patient and family needs. $4,5,8,32,35,37,41$ There are reports that some nurses caring for patients with a KTU have experienced emotional stress and guilt because they believed they failed to implement sufficient 
preventive care. ${ }^{8,34}$ It is vital to raise clinicians' awareness of the unavoidable nature of $\mathrm{KTU}^{7}$ and to set realistic wound care expectations. ${ }^{3,8,16,37}$ Although KTU wound healing and prevention are not possible once the patient is actively dying, ${ }^{3,6,7,16,37}$ this does not imply that this ulcer is a normal part of end of life. ${ }^{2,7}$

\section{Kennedy Terminal Ulcer Health Care Management and Treatment Costs}

In this review, we found no evidence on the specific health care costs associated with the management and treatment of KTU in any health care setting. However, since 2009, the US Centers for Medicare \& Medicaid Services (CMS) accepted that KTUs are unavoidable and should not attract any financial penalties. ${ }^{24}$ This new stance in the United States better reflects the clinical care outcomes for dying patients and contributes to the legitimacy of the existence of KTU. ${ }^{24}$ However, the UK health care policy fails to acknowledge the existence of KTU, but considers all ulcers as preventable PI. ${ }^{43}$

\section{Quality of Life and Psychological Impacts}

Limited information is available on KTU impact on patients' quality of life and psychological well-being. Fourteen studies briefly described some of the impacts of KTU on patients' quality of life and the implications for families and carers. $^{2,4,6,8,19,25,26,30,32-35,37,41}$ An accurate diagnosis of KTU allows clinicians to educate and psychologically prepare patients, families, and carers about the imminence of death $^{8,26}$ and to discuss their end-of-life care choices such as hospice and palliative care..$^{4,8,26,32,35,41}$ Educating families about possible unavoidable skin changes associated with dying ${ }^{6,25,30,34,37}$ can help to alleviate feelings of stress, guilt, and shame if a KTU develops. ${ }^{34,35}$ Setting realistic wound management goals that are situated around comfort and odor control rather than healing is another approach that helps patients, families, and carers understand that despite quality care, KTUs still develop. ${ }^{6,8,25,30,34,35}$ Kennedy terminal ulcers and PIs are painful, so maintaining a patient's comfort and dignity in the days prior to death is paramount. $8,25,33,41$ This requires the input of a specialist team of clinicians. ${ }^{4,25,37}$ Despite this, nurses in a palliative care unit observed many patients with a KTU could not express their feelings of discomfort because of the sedating effect of routine comfort medications. ${ }^{19}$

\section{DISCUSSION}

To our knowledge, this scoping review is the first to identify and collate the breadth of available KTU evidence. Because of the limited KTU evidence, we did not undertake a critical appraisal of the sources ${ }^{13}$; instead, we identified the current knowledge and clinical practice gaps. In the literature, there is no agreement on KTU etiol- ogy, 4,19,33,35,40,43 limited prevalence data across a range of health care settings, ${ }^{5}$ and a lack of assessment and management strategies of KTU including patient education. ${ }^{4,5,7,32,35}$ This dearth of evidence impacts clinicians' ability to deliver targeted end-of-life skin care to their dying patients.

There is contention about the exact etiology of KTU. ${ }^{4,19,33,35,40,43}$ Kennedy terminal ulcers are acknowledged by the NPUAP as unavoidable in some dying patients and are caused by hypoperfusion associated with dying tissues, ${ }^{3}$ although this hypothesis is untested. The ongoing debate about KTUs is emotive, with some blaming their development on health care professionals' delivery of inadequate care. ${ }^{29,31}$ This "blame game" is unhelpful and can negatively impact on the availability of appropriate resources and influence how clinicians and carers deliver this specialized care. ${ }^{4,8,34,37}$ Furthermore, some funding bodies might avoid supporting future KTU research, which could have major implications on progressing our knowledge, assessment, and management of this clinical issue (Table 1).

The provision of quality clinical care to dying patients is paramount. The development of a KTU is distressing for patients, families, and clinicians. ${ }^{29,31,34,35}$ In addition to managing the patients' pain and KTU odor, 6,8,25,30,34,35 clinicians often feel ill-prepared to have open and frank discussions with patients regarding their impending death. ${ }^{8,26}$ Educating clinicians about KTUs will provide them with the knowledge needed to support dying patients and their families and could assist in self-protection against potential guilt.

The first step in all clinical issues is to gain a better understanding regarding the extent of the problem. Recent KTU prevalence data are lacking, with most of the published research involving the presentation of case studies. In the past 35 years, 3 observational studies have been undertaken in adult patients, reporting a KTU prevalence between 5\% and 55.1\%. ${ }^{1,5,19}$ There is a lack of scientific methodologies and analysis in reporting KTU prevalence data in the published literature. ${ }^{3,27}$ Compounding this, it is acknowledged that some KTUs are likely assessed as hospital-acquired PI, ${ }^{2,37}$ increasing the uncertainty of the available prevalence data. Moreover, we found only 1 pediatric KTU study, indicating an urgent need for clinical research area in this population. Based on current evidence, it is impossible to glean accurate estimates of KTU prevalence, and the reliability of existing published data is questionable. Further, current KTU clinical recommendations are based on level 4 evidence. ${ }^{8}$ Hence, there is a compelling need for rigorous clinical research to provide baseline KTU prevalence information in all patient populations across all health care settings.

Arguably, health care professionals' lack of awareness may lead to incorrectly diagnosing KTU as PI. ${ }^{31}$ This has implications for clinical practice relative to care quality, 
the patient and family experience, and health care costs. There appears to be confusion about the assessment and management of KTUs, with discourse related to KTU enmeshed in the discussion of PI. Consequently, this has compounded the confusion and lack of clarity around KTU diagnosis and risk assessment (as distinct from hospitalacquired PI). Currently, a valid KTU assessment tool is lacking, forcing clinicians to use PI assessment tools. Using these assessment tools increases the possibility of an incorrect diagnosis. Accurately diagnosing KTUs allows clinicians to deliver quality patient care that is appropriate $e^{23,31}$ and signals an opportunity to educate patients and families about their end-of-life care choices and approaching death. $4,8,26,32,35,41$ This education can limit unnecessary blame being leveled by families regarding their perceptions of poor nursing care $^{6,8,25,30,34,35}$ and reduce nurses' experience of guilt. ${ }^{8}$ Assembling a specialist team of end-of-life wound care clinicians can support ward-based nurses in delivering appropriate, patient-centered care to their dying patients. ${ }^{4,25,37}$ Finally, in some countries, an accurate KTU diagnosis is justification for financial penalty avoidance, which has legal, financial, and policy implications. This confirms the compelling need for more KTU research to underpin accurate assessment and the development of evidence-based management strategies ${ }^{3,4,36,43}$ (Table 2).

\section{Limitations and Strengths}

Regardless of the review methodology, we acknowledge there are limitations in this work. Scoping reviews take a significant amount of time to complete, and so we recognize that additional relevant KTU sources may have been published since our final database and gray literature searches. We found there is a tendency for KTU information to be embedded within a larger discussion about PI, making it difficult to accurately locate relevant sources. Because of the lack of breadth of the KTU literature, a critical analysis of the strength of the sources was not undertaken $^{13}$; instead, the main aim was to identify current knowledge and clinical practice gaps. ${ }^{\text {? }}$

A major strength of this scoping review is that it has addressed a number of broad questions pertaining to the topic of KTU, a key advantage of this methodological approach. ${ }^{9}$ Finally, this review provides clinicians, researchers, managers, and policy makers with a rigorous narrative of the published KTU literature to inform future research, health care policy, and practice.

\section{RECOMMENDATIONS}

Several recommendations arise from this review that may inform clinical practice, education, and policy. To appreciate the extent of the problem, international research is needed to map KTU prevalence rates in acute care, hospice, and nursing home settings. The management and treatment of KTU also require further investigation, so that targeted holistic patient-centered strategies become part of ongoing care. The accurate assessment of KTUs is achieved through the development of specialized end-of-life risk assessment tools. In addition, there is a need to raise clinicians' awareness of KTU through ongoing education, with both underpinned by additional research. These strategies may contribute toward improving patients' end-of-life care, reducing financial penalties, and may influence current health care and organizational policies.

\section{GONGLUSION}

This scoping review revealed significant knowledge and clinical practice gaps. Kennedy terminal ulcers are unavoidable, so appropriate management, including patient education, helps dying patients maintain some quality of life. Increasing our understanding of KTUs will allow clinicians to provide targeted clinical care and educate patients about their end-of-life care choices. For health care organizations, the accurate assessment of KTUs may contribute to avoiding unwarranted financial penalties. Further KTU research is urgently needed to provide clinicians, patients, and families with evidence to inform future clinical care.

\section{References}

1. Kennedy KL. The prevalence of pressure ulcers in an intermediate care facility. Decubitus. 1989;2(2):44-45.

2. Kennedy-Evans K. Understanding the Kennedy terminal ulcer. Ostomy Wound Manage. 2009;55(9):6-6.

3. Alvarez OM, Brindle CT, Langemo D, et al. The VCU pressure ulcer summit: the search for a clearer understanding and more precise clinical definition of the unavoidable pressure injury. $J$ Wound Ostomy Continence Nurs. 2016;43(5):455-463.

4. Vera R. Literature Review of Kennedy Terminal Ulcers: Identification, Diagnosis, Nursing Goals, and Interventions. Salem, MA: Health and Human Services, Salem State University; 2014.

5. Nesovic AV. Kennedy Terminal Ulcer: A Retrospective Chart Review of Ulcers in the Hospice Setting and Educating Providers and Nurses on the Importance of Skin Changes at Life's End. Bozeman, MT: Nursing, Montana State University; 2016.

6. Lepak V. Avoidable \& inevitable? Skin failure: the Kennedy terminal lesion. J Legal Nurse Consult. 2012;23(1):24-27.

7. Beldon P. Skin Changes at Life's End (SCALE): a consensus document. Wounds UK. 2010;6(1):169-170.

8. Shepard J. Hospice Nurses' Experience of Caring for the Elderly With Wounds at the End-of-life. Greenville, NC: Nursing, East Carolina University; 2015.

9. Arksey H, O'Malley L. Scoping studies: towards a methodological framework. Int J Soc Res Methodol. 2005;8(1):19-32.

10. Peters MD, Godfrey CM, Khalil H, McInerney P, Parker D, Soares C. Guidance for conducting systematic scoping reviews. Int J Evid Based Healthc. 2015;13(3):141-146.

11. Takashima M, Ray-Barruel G, Keogh S, Rickard C. Randomised controlled trials in peripheral vascular access catheters: a scoping review. Vasc Access. 2015;1(2):10-37.

12. Kunst E, Henderson A, Johnston A. A scoping review of the use and contribution of simulation in Australian undergraduate nurse education. Clin Simul Nurs. 2018;19:17-29. 
13. Tricco AC, Lillie E, Zarin W, et al. PRISMA Extension for Scoping Reviews (PRISMA-ScR): checklist and explanation. Ann Intern Med. 2018;169(7):467-473.

14. Independent Hospital Pricing Authority. Palliative care. https:// www.ihpa.gov.au/what-we-do/palliative-care. Accessed September 17, 2018

15. Batchelor NH. Palliative or hospice care? Understanding the similarities and differences. Rehabil Nurs. 2010;35(2):60-64.

16. Horn J, Irion GL. The integument: current concepts in care at end of life. J Acute Care Phys Ther. 2014;5(1):11-17.

17. Sanford AM, Orrell M, Tolson D, et al. An international definition for "nursing home". J Am Med Dir Assoc. 2015;16(3):181-184.

18. Moher D, Liberati A, Tetzlaff J, Altman DG. Preferred reporting items for systematic reviews and meta-analyses: the PRISMA statement. PLoS Med. 2009;6(7):e1000097.

19. Brennan MR, Trombley K. Kennedy terminal ulcers-a palliative care unit's experience over a 12-month period of time. WCET J. 2010;30(3):20-22.

20. Trombley K, Brennan MR, Thomas L, Kline M. Prelude to death or practice failure? Trombley-Brennan terminal tissue injuries. Am J Hosp Palliat Med. 2012;29(7):541-545.

21. Langemo DK, Brown G. Skin fails too: acute, chronic, and endstage skin failure. Adv Skin Wound Care. 2006;19(4):206-212.

22. Merugu G, Rosenzweig A. Wound care. In: Fenstemacher PA, Winn P, eds. Post-acute and Long-term Medicine: A Pocket Guide. 2nd ed. Philadelphia, PA: Springer International Publishing, 2016:217-234.

23. Centers for Medicare \& Medicaid Services. CMS updates: Kennedy terminal ulcer, mucosal pressure ulcers, and revised IRF-PAI. Adv Skin Wound Care. 2013;26(10):444-444.

24. Centers for Medicare \& Medicaid Services. CMS recognizes Kennedy terminal ulcer in long-term care hospitals. Ostomy Wound Manage. 2013;59(8):42-42.

25. Graves ML, Sun V. Providing quality wound care at the end of life. J Hosp Palliat Nurs. 2013;15(2):66-74.

26. Buscemi C. Kennedy Terminal Ulcer: A Case Study. Presented at the Southeast Region Wound Ostomy and Continence Nurses Society Conference; September 10-12, 2015; Florida.

27. Lutz J, Schank J. The Kennedy Terminal Ulcer: Twenty Years
Later. Presented at the 41st Annual Wound Ostomy and Continence Nurses Conference; 2009; St Loius, MO.

28. Martin K. Understanding the Kennedy Terminal Ulcer. 2014. http://www.kennedyterminalulcer.com/. Accessed January 8, 2018.

29. Miller MS. The death of the Kennedy terminal ulcer. J Am Coll Clin Wound Spec. 2017, 8(1-3):44-46.

30. Miner KJ. Discharge to hospice: a Kennedy terminal ulcer case report. J Am Coll Clin Wound Spec. 2009;1(3):84-85.

31. Olshansky K. "Kennedy terminal ulcer" and "skin failure," where are the data? J Wound Ostomy Continence Nurs. 2010; 37(5):466.

32. Reitz M, Schindler CA. Pediatric Kennedy terminal ulcer. $J$ Pediatr Health Care. 2016;30(3):274-278

33. Rivera J, Stankiewicz M. A review of clinical incidents: skin failure in the dying patient. J Stomal Ther Aust. 2018;38(1):12-14.

34. Schank J. Notes on practice: elder abuse or Kennedy terminal ulcer? Ostomy Wound Manage. 2013.

35. Schank JE. Kennedy terminal ulcer: the "ah-ha!" moment and diagnosis. Ostomy Wound Manage. 2009;55(9):40-44.

36. Sibbald RG, Krasner DL, Lutz J. SCALE: Skin Changes at Life's End: final consensus statement: October 1, 2009. Adv Skin Wound Care. 2010;23(5):225-236. quiz 237-238.

37. Yastrub DJ. Pressure or pathology: distinguishing pressure ulcers from the Kennedy terminal ulcer. J Wound Ostomy Continence Nurs. 2010;37(3):249-250.

38. Brennan M. Trombley-Brennan terminal tissue injuries. In: National Pressure Ulcer Advisory Panel 2017. 2017.

39. Levine JM. Unavoidable pressure injuries, terminal ulceration, and skin failure: in search of a unifying classification system. Adv Skin Wound Care. 2017;30(5):200-202.

40. Beldon P. Skin Changes at Life's End: SCALE ulcer or pressure ulcer? Br J Community Nurs. 2011;16(10):491-494.

41. Hampton S. Skin changes and skin care for people with diabetes at the end of life. J Diabetes Nurs. 2016;20(9):336-339.

42. Sarabia-Cobo CM. Poly ulceration patient terminal: Kennedy terminal ulcer (KTU). J Palliat Care Med. 2017;7(1):100297.

43. Stephen-Haynes J. Pressure ulceration and palliative care: prevention, treatment, policy and outcomes. Int J Palliat Nurs. 2012;18(1):9-16. 